Aim of the study: Our aim was to analyse the feasibility of white blood cell and platelet counts along with their ratios as a prognostic factor in patients who underwent surgery for ovarian mass.

Material and methods: We retrospectively studied the patients admitted in the Department of Gynaecology due to adnexal mass. The potential association of the neutrophil-to-lymphocyte-ratio (NLR), neutrophil-to-monocyte (NMR), platelet-to-lymphocyte (PLR), lymphocyte-to-monocyte (LMR), monocyte-to-platelet, and malignancy was evaluated.

Results: Patients with malignant tumours were found with significantly higher ratios of NLR $(p<0.001)$ and PLR $(p<0.001)$ and lower LMR ratio $(p<0.001)$ compared to those with benign tumours. Furthermore, higher lymphocyte count $(p=0.04)$ and platelet count $(p=0.004)$ were found in cancer patients when compared with borderline tumours. No significant variations were detected regarding white blood cell count $(p=0.238)$, NMR ratio $(p=0.28)$, platelet-to-neutrophil ratio $(p=0.12)$, and platelet-to-monocyte ratio $(p=0.34)$.

Conclusions: Inflammation biomarker ratios can easily and inexpensive assist in distinguishing malignant ovarian tumours from benign ones.

Key words: pelvic tumours, inflammation biomarkers, differential diagnosis.

Contemp Oncol (Pozn) 2021; 25 (1): 7-11 DOI:https://doi.org/10.5114/wo.2021.105072

\section{The role of inflammation}

\section{biomarkers in differential diagnosis of pelvic tumours of ovarian origin: a single-centre observational study}

\author{
Victoria Psomiadou ${ }^{1}$, Anastasia Prodromidou ${ }^{2}$, Elpis Galati ${ }^{1}$, loannis D. Kostakis ${ }^{3}$, \\ Athanasios Maliaros ${ }^{1}$, Ioannis Mamais ${ }^{1}$, Nikolaos Blontzos ${ }^{1}$, Fotios Lefkopoulos ${ }^{1}$, \\ Christos lavazzo $^{1}$, George Vorgias ${ }^{1}$
}

${ }^{1}$ Department of Gynaecological Oncology, Metaxa Memorial Cancer Hospital, Piraeus, Greece

${ }^{2}$ Department of Surgical Oncology, Metaxa Memorial Cancer Hospital, Piraeus, Greece 'Department of Transplantation, Guy's Hospital, Guy's and St Thomas' NHS Foundation Trust, London, UK

\section{Introduction}

Ovarian cancer $(\mathrm{OC})$ constitutes the $5^{\text {th }}$ most common female cancer worldwide. According to the statistics of 2012, 65,900 and 86,000 women in economically developed and developing countries, respectively, died due to the disease [1]. During the last decades, OC has remained the most lethal malignancy among women worldwide, with a median overall survival which barely reaches 30\% [2]. Histological grade and staging of the disease, number of resected lymph nodes, increased values of tumour markers such as human epididymis protein 4 (HE4) and cancer antigen 125 (CA125), as well as the risk of malignancy index and the risk of malignancy algorithm have been demonstrated to have high predictive and prognostic significance in patients with OC $[3,4]$. However their application in clinical practice is limited due to the lack of standardization and high cost [5]. Furthermore, recently, an increasing interest in the association between inflammation and cancer has been widely expressed [5]. Inflammation has been reported as a significant risk factor that predisposes to alternation of tissues from premalignant to malignant. In that setting, the inflammation process can cause cell turnover, and the created microenvironment can often lead to an increase in DNA damage or mutation [6].

Neutrophils, lymphocytes, monocytes, platelets, and their ratios, specifically the neutrophil-to-lymphocyte ratio (NLR) and platelet-to-lymphocyte ratio $(P L R)$, have been proposed as useful biomarkers in the diagnosis and management of various cancers, including endometrial, cervical, pancreatic, gastric, and breast cancer [7-10]. The application of NLR and PLR ratios as a screening test in patients with adnexal tumours has been investigated by several studies $[11,12]$. Nonetheless, the clinical utility and the association of these biomarkers with prognosis in patients with pelvic tumours has yet to be elucidated.

In this study we aimed to evaluate the utility of inflammatory ratios as a prognostic factor in women undergoing surgery for an ovarian mass.

\section{Material and methods}

Study population

A retrospective analysis of patients who were admitted in the Department of Gynaecology of the Metaxa Memorial Cancer Hospital of Piraeus from May 2016 to May 2018 due to adnexal mass was performed. The Institutional Review Board or Ethics Committee approved our research protocol before 
the initiation of the study, and all human participants and their parents gave written/verbal informed consent. All women were preoperatively evaluated with ultrasonography (US) and/or computer tomography and/or magnetic resonance. All of them underwent surgery with the intention to treat or to stage their disease. Levels of preoperative neutrophils, lymphocytes, monocytes, and platelets were measured and NLR, PLR, lymphocyte-to-monocyte $(L M R)$, and platelet-to-monocyte ratio (PMR) were then calculated. The pathology department of our hospital conducted the histopathological analysis and characterized the resected specimens as malignant, borderline, or benign. Finally, the examined ratios were correlated to the histological type of the resected masses. A sub-analysis according to stage and grade of malignant tumours was also performed.

\section{NLR, PLR, LMR, and PMR calculation}

The absolute neutrophil count was divided by the absolute lymphocyte count to determine the NLR; Respectively, PLR was determined by dividing the absolute platelet count by the absolute lymphocyte count, LMR by dividing the absolute lymphocyte count by the absolute monocyte count, and PMR by dividing the absolute monocyte count by the absolute platelet count.

\section{Statistical analysis}

The Shapiro-Wilk test was used to estimate the normality of data distribution. Student's t-test, Welch test, or Mann-Whitney $U$ test were performed as appropriate to perform comparisons between 2 groups, while the comparisons among 3 groups were performed using the analysis of variance (ANOVA) with the Bonferroni correction or the Kruskal-Wallis test with the Bonferroni correction, as appropriate. Correlations between 2 continuous variables were assessed using Pearson's correlation coefficient or Spearman's rank correlation coefficient, as appropriate. To identify the optimal cut-off points for parameters that provided significant results, we used a combination of the receiver operating characteristic curve and Youden's J statistic. Sensitivities and specificities were calculated based on these optimal cut-off points. All the tests were 2-tailed. Results were considered statistically significant if the $p$-value was less than 0.05. The statistical analysis was performed with the $25^{\text {th }}$ edition of the Statistical Package for the Social Sciences (IBM, Armonk, NY, USA).

\section{Results}

One hundred and seventy-nine women presenting with ovarian tumours were included in our study. One hundred and twenty-four had benign tumour (69.3\%), 9 had borderline tumour (5\%), and 46 had malignant tumour (25.7\%). The mean age was 53.5 years (SD: 15), and the median age was 53 years (min-max: 20-86). Ninety-seven women were premenopausal, and 82 women were postmenopausal. Among patients with malignant disease, 9 were diagnosed with stage I disease, 4 with stage II, 32 with stage III, and 3 with stage IV.

\section{Comparison among tumour groups}

Borderline and malignant tumours were more frequent in postmenopausal women than in premenopausal women [premenopausal: benign: 76/97 (78.4\%), borderline: 3/97 (3.1\%), malignant: 18/97 (18.6\%), postmenopausal: benign: 48/82 (58.5\%), borderline: 6/82 (7.3\%), malignant: 28/82 (34.1\%) $(p=0.015)$ ]. With regards to leukocyte and platelet indices, malignant tumours had higher neutrophil count (median: 5432, min-max: 2042-9219 vs. median: 4326.5, min-max: 2169-15306, $p=0.013$ ), monocyte count (median: 470.5, min-max: 269-828 vs. median: 402, min-max: 169 vs. $1574, p=0.042$ ), platelet count (median: 307,000 , min-max: 149,000-884,000 vs. median: 265,000, min-max: 147,0004,660,000, $p<0.001$ ), NLR (median: 2.8, min-max: 1.2-17.4 vs. median: 2.1, min-max: 0.8-8.8, $p<0.001$ ), and PLR (median: 184.2, min-max: 76.1-502.8 vs. median: 128.4, min-max: 35.6-332.1, $p<0.001$ ), and lower lymphocyte count (median: 1786.5, min-max: 458-3887 vs. median: 2176, min-max: 972-5219, $p=0.004$ ) and LMR (median: 3.8, min-max: 1.39.7 vs. median: 5 , min-max: 1.7-11.4, $p<0.001$ ) when compared with benign tumours. Moreover, malignant tumours had higher lymphocyte count (median: 1786.5, min-max: 458-3887 vs. median: 1652, min-max: 481-2343, $p=0.04$ ) and platelet count (median: 307000, min-max: 149,000884,000 vs. median: 236,000, min-max: 162,000-300,000, $p=0.004)$ when compared with borderline tumours. On the other hand, no significant variations were detected regarding white blood cell count $(p=0.238)$, NLR $(p=0.28)$, PLR $(p=0.12)$, and PMR $(p=0.34)$.

\section{Differentiating malignant from benign/borderline tumours}

Leukocyte and platelet indices provided mediocre results. In particular, the AUC for neutrophil count was 0.638 (SE: $0.05,95 \% \mathrm{Cl}: 0.54-0.736, p=0.005)$, the AUC for lymphocyte count was 0.644 (SE: $0.046,95 \%$ Cl: 0.555-0.734, $p=0.004$ ), the AUC for monocyte count was 0.627 (SE: $0.047,95 \% \mathrm{Cl}: 0.535-0.72, p=0.01)$, the AUC for platelet count was 0.703 (SE: $0.048,95 \% \mathrm{Cl}: 0.609-0.798$, $p<0.001$ ), the AUC for NLR was 0.69 (SE: $0.046,95 \% \mathrm{Cl}$ : $0.6-0.779, p<0.001$ ), the AUC for LMR was 0.712 (SE: $0.045,95 \% \mathrm{Cl}: 0.623-0.801, p<0.001$ ), and the AUC for PLR was 0.759 (SE: $0.044,95 \% \mathrm{Cl}: 0.674-0.845, p<0.001$ ). The optimal cut-off points for identifying malignancy were the following: neutrophil count: $\geq 6184.5$ (sensitivity: 41.3\%, specificity: $85 \%$ ), lymphocyte count: $\leq 2303$ (sensitivity: $89.1 \%$, specificity: $40.6 \%$ ), monocyte count: $\geq 452.5$ (sensitivity: $65.2 \%$, specificity: $63.9 \%$ ), platelet count: $\geq 284,500$ (sensitivity: 69.6\%, specificity: 67.7\%), NLR: $\geq 2.4$ (sensitivity: $67.4 \%$, specificity: $65.4 \%$ ), LMR: $\leq 4.55$ (sensitivity: $71.7 \%$, specificity: $65.4 \%$ ), and PLR: $\geq 181.1$ (sensitivity: $60.9 \%$, specificity: $83.5 \%$ ).

\section{Differentiating malignant/borderline from benign tumours}

Leukocyte and platelet indices provided mediocre results. In particular, the AUC for neutrophil count was 0.629 (SE: $0.047,95 \% \mathrm{Cl}$ : 0.537-0.721, $p=0.006$ ), the AUC for lymphocyte count was 0.675 (SE: $0.042,95 \%$ Cl: $0.592-$ 
Table 1. Values of the examined biomarkers and statistical significance of comparison among the groups

\begin{tabular}{|c|c|c|c|c|c|}
\hline & $\begin{array}{l}\text { Benign } \\
(n=124)\end{array}$ & $\begin{array}{l}\text { Borderline } \\
\quad(n=9)\end{array}$ & $\begin{array}{l}\text { Malignant } \\
(n=46)\end{array}$ & $\begin{array}{c}p \text {-value } \\
\text { malignant vs. benign }\end{array}$ & $\begin{array}{c}p \text {-value malignant } \\
\text { vs. borderline }\end{array}$ \\
\hline \multicolumn{6}{|c|}{ Neutrophil count } \\
\hline Mean & 4751 & 5582 & 4950 & \multirow[t]{5}{*}{0.013} & \multirow[t]{4}{*}{0.238} \\
\hline Median & 4436 & 5139 & 5432 & & \\
\hline SD & 1978.29 & 1619.73 & 1957.55 & & \\
\hline Range & 13137 & 5134 & 7177 & & \\
\hline \multicolumn{5}{|c|}{ Lymphocyte count } & \\
\hline Mean & 2190.94 & 1556.11 & 1802.95 & \multirow[t]{4}{*}{0.004} & \multirow[t]{4}{*}{0.04} \\
\hline Median & 2176 & 1652 & 1786.5 & & \\
\hline SD & 687.66 & 613.90 & 612.85 & & \\
\hline Range & 4247 & 1862 & 3429 & & \\
\hline \multicolumn{6}{|c|}{ Monocyte count } \\
\hline Mean & 443.47 & 400.55 & 496.02 & \multirow[t]{5}{*}{0.042} & \multirow[t]{4}{*}{ NS } \\
\hline Median & 402 & 362 & 470.5 & & \\
\hline SD & 179.96 & 154.29 & 147.66 & & \\
\hline Range & 1405 & 489 & 559 & & \\
\hline \multicolumn{5}{|c|}{ Platelet count } & \\
\hline Mean & 271,911.29 & $235,222.22$ & $344,565.21$ & \multirow[t]{4}{*}{$<0.001$} & \multirow[t]{4}{*}{0.004} \\
\hline Median & 265,000 & 236,000 & 307,000 & & \\
\hline SD & $70,079.51$ & $42,313.64$ & $131,180.22$ & & \\
\hline Range & 319,000 & 138,000 & 735,000 & & \\
\hline \multicolumn{6}{|l|}{ NLR } \\
\hline Mean & 2.34 & 4.00 & 3.62 & \multirow[t]{4}{*}{$<0.001$} & \multirow[t]{4}{*}{0.28} \\
\hline Median & 2.14 & 3.18 & 2.80 & & \\
\hline SD & 1.18 & 2.69 & 2.64 & & \\
\hline Range & 8.01 & 7.91 & 16.23 & & \\
\hline \multicolumn{6}{|l|}{ PLR } \\
\hline Mean & 134.59 & 180.68 & 210.61 & \multirow[t]{5}{*}{$<0.001$} & \multirow[t]{4}{*}{0.12} \\
\hline Median & 128.36 & 152.56 & 184.22 & & \\
\hline SD & 50.47 & 88.04 & 98.64 & & \\
\hline Range & 296.48 & 251.99 & 426.70 & & \\
\hline \multicolumn{5}{|l|}{ LMR } & \\
\hline Mean & 5.32 & 4.17 & 3.94 & \multirow[t]{4}{*}{$<0.001$} & \multirow[t]{4}{*}{ NS } \\
\hline Median & 5.0 & 3.35 & 3.8 & & \\
\hline SD & 1.8 & 1.91 & 1.78 & & \\
\hline Range & 9.66 & 5.27 & 8.41 & & \\
\hline
\end{tabular}

SD - standard deviation

$0.758, p<0.001)$, the AUC for monocyte count was 0.593 (SE: $0.046,95 \% \mathrm{Cl}: 0.502$ vs. $0.684, p=0.048$ ), the AUC for platelet count was 0.639 (SE: $0.048,95 \%$ Cl: 0.545 vs. 0.732 , $p=0.003$ ), the AUC for NLR was 0.708 (SE: $0.043,95 \% \mathrm{Cl}$ : $0.624-0.792, p<0.001)$, the AUC for LMR was 0.716 (SE: $0.043,95 \% \mathrm{Cl}: 0.632-0.801, p<0.001)$, and the AUC for PLR was 0.753 (SE: $0.041,95 \% \mathrm{Cl}: 0.672-0.834, p<0.001)$. The optimal cut-off points for identifying malignant/borderline tumours were the following: neutrophil count: $\geq 5136.5$ (sensitivity: 58.2\%, specificity: 67.7\%), lymphocyte count: $\leq 2303$ (sensitivity: 89.1\%, specificity: 42.7\%), monocyte count: $\geq 452.5$ (sensitivity: $60 \%$, specificity: $63.7 \%$ ), plate- 
let count: $\geq 284,500$ (sensitivity: $60 \%$, specificity: $66.1 \%$ ), NLR: $\geq 2.52$ (sensitivity: 63.6\%, specificity: 71.8\%), LMR: $\leq 3.94$ (sensitivity: $56.4 \%$, specificity: $79.8 \%$ ), and PLR: $\geq 142.7$ (sensitivity: $78.2 \%$, specificity: $64.5 \%$ ) (Table 1 ).

\section{Discussion}

Systemic inflammatory response has been demonstrated to be associated with cancer progression and survival, and inflammation markers have been explored extensively as prognostic factors [10], with NLR specifically being suggested by many studies as significant [13]. Blood cell counts and their ratios, such as NLR, PLR, and LMR, are considered markers indicating systemic inflammation [14]. However, the exact role of those parameters in predicting the oncological outcome has not yet been elucidated. Our study sought to report a longitudinal comparison of 6 systemic inflammation-based prognostic ratios, and to evaluate their role in differentiating malignant from benign and borderline ovarian tumours. Our study suggests a strong association between the majority of the investigated biomarkers (NLR, PLR, and LMR) and detection of malignancy in patients with adnexal masses. No difference was found for NLR, PLR, and PMR ratios among patients with malignant and borderline disease.

Neutrophils are the white blood cells that control the inflammatory response of normal cells against cancer mutations. The cytokines that they secrete, such as interleukins and vascular endothelial growth factor, are responsible for activating various molecular pathways that interact with normal tissue cells and other inflammatory cells, such as platelets and lymphocytes, and prolong the inflammatory process [15]. Activated neutrophils block the cytotoxic activity of T-cell lymphocytes, resulting in an immunosuppressive tumour environment [16]. In this micro-environment, a sequence of molecular interactions is responsible for tumour spread, angiogenesis, and metastasis to distant sites [17].

Lymphocytes have been proposed to play a well-established role in cytotoxic cell death and cytokine cascade, preventing proliferation and metastatic activity of malignant cells. Additionally, lymphocytes known as tumour infiltrating lymphocytes have been detected in the tumour site and play an important role in killing tumour cells and preventing tumour genesis and metastasis [2, 18]. They have been described in many cancers, including OC, and have been associated with good prognosis [15].

Monocytes, on the other hand, are transformed into macrophages when attracted by factors that tumour cells produce and have cytotoxic activity, destroying cancer cells. However, tumour-associated macrophages also have other different functions that trigger chronic inflammation and secrete cytokines that enhance oncogenesis and metastasis by creating an immunosuppressive environment around the tumour [1]. Therefore, when the monocyte count of the peripheral blood is higher than normal in a cancer patient, it is considered an adverse prognostic factor [1]

The role of platelets in tumour proliferation has been widely studied. Platelets are activated by the chronic in- flammatory response and secrete TGF-, which attracts neutrophils to the tumour site, which, as mentioned above, help tumour growth $[1,15]$. Activated platelets trigger other mechanisms, such as thrombosis and angiogenesis and, eventually, metastasis [15]. An increased platelet number in the peripheral blood is described in many cancers, including ovarian cancer: more than a fifth of patients with epithelial ovarian cancer (EOC) have thrombocytosis [18]. Thrombocytosis at the time of diagnosis is related to poor survival and resistance to chemotherapy [18].

NLR constitutes an inflammation marker $[16,18]$, possibly able to differentiate malignant from benign ovarian tumours [19]. High NLR score refers to a high neutrophil count and a low lymphocyte count in the patient's serum. Yildirim et al. found a sensitivity and specificity of $65 \%$ and $72 \%$, respectively, for the early diagnosis of EOC [11]. This ratio was found to be higher in advanced-stage $O C$ and high histological grade $[19,20]$. An elevated NLR score has been related to an unfavourable response to chemotherapy [20]. According to Miao et al., elevated NLR can predict chemoresistance with a sensitivity of $75 \%$ and a specificity of $80 \%$ [20]. Thus, NLR and prognosis and overall survival of EOC are closely related. Elevated NLR values have been linked to poor prognosis, overall survival, and progression-free survival $[2,16]$. Ashrafganjoei et al. demonstrated that NLR could predict surgical outcome in EOC patients, with a reported sensitivity of $74 \%$ and of specificity $58 \%$. These findings are in harmony with those of our analysis, because NLR and PLR also showed diagnostic sensitivity in OC (AUC = 0.69, AUC = 0.759). Interestingly, these results are encouraging compared to those of other studies analysing the same ratios in non-gynaecological malignancies. Machairas et al. concluded that white blood cell and platelet counts cannot assist in diagnosing early thyroid cancer [10], while other studies examining a potential link between the inflammation biomarkers NLR and PLR and neoplasms of the gastrointestinal track, although demonstrating a significant diagnostic value, lack a detailed discussion of specificity and sensitivity [9, 14].

PLR can also predict malignancy of an adnexal tumour when assessed preoperatively (sensitivity $74 \%$, specificity $67 \%$ ) whereas elevated PLR levels can negatively affect the survival of patients with EOC [17] . Increased levels of PLR represent thrombocytosis in the peripheral blood and consequently can indicate resistance to chemotherapy (sensitivity $60 \%$ and specificity $85 \%$ ) [21].

LMR can also serve as a prognostic factor for ovarian malignancies [1]. Low LMR scores are related to a high monocyte count, and therefore an unfavourable prognosis and survival [1]. This association has been described in many cancers, such as Hodgkin's lymphoma and ovarian cancer. Unfortunately, there are not many studies in the literature examining the clinical utility of LMR. NLR and PLR have been demonstrated as useful predictors of malignancy. Our analysis highlights that additional ratios such as LMR can possibly be confirmed as prognostic biomarkers in ovarian cancer. However, the application of LMR as a screening tool in the general population has not been validated yet. Additionally, there are some further limitations before interpreting our outcomes that need to be addressed. First of all, 
our study was performed during a limited period between 2016 and 2018. All gynaecological oncology operations were performed by our team led by 3 different consultants based on MDT decisions and international guidelines. For this reason, and because of the small period of the study (3 years), no significant change could be identified between changes over time or any therapy-dependent changes. Another weakness of the article is that our results cannot be clearly clinically useful yet.

\section{Conclusions}

Inflammation biomarkers can serve as significant indicators of malignancy in patients with ovarian tumours. The examined ratios depict the status of the immune system of the patient and are therefore potentially able to define the prognosis of ovarian malignancy. They comprise an easily calculated and inexpensive test that can be easily utilized in everyday clinical practice. Further research is warranted in this field to determine the predictive utility of the inflammatory biomarkers so as to routinely introduce them into clinical practice.

The authors declare no conflict of interest.

\section{References}

1. Yang WL, Lu Z, Bast RC. The role of biomarkers in the management of epithelial ovarian cancer. Expert Rev Mol Diagn 2017; 17: 577 591.

2. Anton C, Carvalho FM, Oliveira EI, Maciel GA, Baracat EC, Carvalho JP. A comparison of CA125, HE4, risk ovarian malignancy algorithm (ROMA), and risk malignancy index (RMI) for the classification of ovarian masses. Clinics (Sao Paulo) 2012; 67: 437-441.

3. Bast RC, Jr., Skates S, Lokshin A, Moore RG. Differential diagnosis of a pelvic mass: improved algorithms and novel biomarkers. International journal of gynecological cancer. Int J Gynecol Cancer 2012; 22: S5-8.

4. Peek RM Jr., Mohla S, DuBois RN. Inflammation in the genesis and perpetuation of cancer: summary and recommendations from a national cancer institute-sponsored meeting. Cancer Res 2005; 65: 8583-8586.

5. Costantini S, Capone F, Guerriero E, Castello G. An approach for understanding the inflammation and cancer relationship. Immunol Lett 2009; 126: 91-92.

6. Bacanakgil BH, Kaban I, Unal F, Guven R, Sahin E, Yildirim SG. Predictive value of hematological inflammatory markers in endometrial neoplasia. Asian Pac J Cancer Prev 2018; 19: 1529-1532.

7. Wang L, Jia J, Lin L, et al. Predictive value of hematological markers of systemic inflammation for managing cervical cancer. Oncotarget 2017; 8: 44824-32.

8. Zhang Y, Lu JJ, Du YP, Feng CX, Wang LQ, Chen MB. Prognostic value of neutrophil-to-lymphocyte ratio and platelet-to-lymphocyte ratio in gastric cancer. Medicine 2018; 97: e0144.

9. Machairas N, Kostakis ID, Prodromidou A, et al. Trends in white blood cell and platelet indices in a comparison of patients with papillary thyroid carcinoma and multinodular goiter do not permit differentiation between the conditions. Endocri Res 2017; 42: 311-317.

10. Vural F, Aka N, Ertas S, Kose G, Tufekci EC. The ovarian cancers in geriatric population: the validity of inflammatory markers, malignancy risk indices 1, 2, 3, 4, and CA-125 levels in malignancy discrimination of adnexal masses. Eur J Gynaecol Oncol 2016; 37: 846-851.

11. Yildirim MA, Seckin KD, Togrul C, et al. Roles of neutrophil/lymphocyte and platelet/lymphocyte ratios in the early diagnosis of malignant ovarian masses. Asian Pac J Cancer Prev 2014; 15: 68816885.

12. Zhu Y, Zhou S, Liu Y, Zhai L, Sun X. Prognostic value of systemic inflammatory markers in ovarian Cancer: a PRISMA-compliant meta-analysis and systematic review. BMC Cancer 2018; 18: 443.

13. Zhou ZQ Pang S, Yu XC, et al. Predictive values of postoperative and dynamic changes of inflammation indexes in survival of patients with resected colorectal cancer. Curr Med Sci 2018; 38: 798808.

14. Singel KL, Segal BH. Neutrophils in the tumor microenvironment: trying to heal the wound that cannot heal. Immunol Rev 2016; 273 : 329-343.

15. Ethier JL, Desautels DN, Templeton AJ, Oza A, Amir E, Lheureux S. Is the neutrophil-to-lymphocyte ratio prognostic of survival outcomes in gynecologic cancers? A systematic review and meta-analysis. Gynecol Oncol 2017; 145: 584-594.

16. Zhao Z, Zhao X, Lu J, Xue J, Liu P, Mao H. Prognostic roles of neutrophil to lymphocyte ratio and platelet to lymphocyte ratio in ovarian cancer: a meta-analysis of retrospective studies. Arch Gynecol Obstet 2018; 297: 849-857.

17. Baert T, Van Camp J, Vanbrabant L, et al. Influence of CA125, platelet count and neutrophil to lymphocyte ratio on the immune system of ovarian cancer patients. Gynecol Oncol 2018; 150: 31-37.

18. Prodromidou A, Andreakos P, Kazakos C, Vlachos DE, Perrea D, Pergialiotis $\mathrm{V}$. The diagnostic efficacy of platelet-to-lymphocyte ratio and neutrophil-to-lymphocyte ratio in ovarian cancer. Inflamm Res 2017; 66: 467-475.

19. Huang QT, Zhou L, Zeng WJ, et al. Prognostic significance of neutrophil-to-lymphocyte-ratio in ovarian cancer: a systematic review and meta-analysis of observational studies. Cell Physiol Biochem 2017; 41: 2411-2418.

20. Miao Y, Yan Q, Li S, Li B, Feng Y. Neutrophil to lymphocyte ratio and platelet to lymphocyte ratio are predictive of chemotherapeutic response and prognosis in epithelial ovarian cancer patients treated with platinum-based chemotherapy. Cancer Biomark 2016; 17: 33-40.

21. Ashrafganjoei T, Mohamadianamiri M, Farzaneh F, Hosseini MS, Arab M. Investigating preoperative hematologic markers for prediction of ovarian cancer surgical outcome. Asian Pac J Cancer Prev 2016; 17: 1445-1448.

\section{Address for correspondence}

\section{MD, MSc Victoria Psomiadou}

Department of Gynaecological Oncology

51 Botasi St.

18537 Piraeus, Greece

Piraeus, Greece

e-mail: psomiadouvictoria@gmail.com

Submitted: 10.09 .2020

Accepted: 4.12.2020 